Proyecciones Journal of Mathematics

Vol. 32, No 1, pp. 47-60, March 2013.

Universidad Católica del Norte

Antofagasta - Chile

\title{
On generalized preopen sets
}

\author{
Talal Al-Hawary \\ Yarmouk University, Jordan \\ Received : December 2011. Accepted : December 2012
}

\begin{abstract}
In this paper, the notion of generalized preopen compactness is introduced and connections to other several types of compactness are discussed. In addition, new separation axioms are established.
\end{abstract}

Keywords : Generalized preopen set, generalized preopen compactness.

2004 AMS Subject Classification. 54C08, $54 \mathrm{HO}$. 


\section{Introduction}

Let $(X, \tau)$ be a topological space (or simply, a space) and $A \subseteq X$. Then $A$ is $\alpha$-open or $\alpha$-set [14, 12] (resp., semi-open [2], semi-closed [2], preopen [11], preclosed [11], semi-preopen [2], generalized closed [9]) if $A \subseteq$ $\operatorname{int}(c l(\operatorname{int}(A)))($ resp., $A \subseteq c l(\operatorname{int}(A)), \operatorname{int}(c l(A)) \subseteq A, A \subseteq \operatorname{int}(c l(A))$, $c l(\operatorname{int}(A)) \subseteq A, A \subseteq \operatorname{cl}(\operatorname{int}(\operatorname{cl}(A))), \operatorname{cl}(A) \subseteq U$, for every open set $U$ containing $A$ ), where $\operatorname{int}()$ and $\operatorname{cl}()$ are the interior and closure operations, respectively. $A$ is regular-open if $A=\operatorname{int}(\operatorname{cl}(A))[16]$. Complements of regular-open sets are called regular-closed. $A$ is semi-regular [11] if it is both semi-open and semi-closed. $A$ is interior closed [7] if $\operatorname{int}(A)$ is semiclosed. $A$ is an $A$-set [16] if $A=U \cap C$, where $U$ is an open set and $C$ is a regular closed set. It is known that an A-set is semi-open [16]. A is a B-set [4] if $A=U \cap C$ where $U$ is an open set and $C$ is semi-closed. $A$ is locally closed if $A$ is open in its closure [4]. $A$ is an $A B$ - set [4] if $A=U \cap C$, where $U$ is open and $C$ is semi-regular. Equivalently, $A$ is an locally closed set if $A=U \cap C$, where $U$ is open and $C$ is closed. Clearly every A-set is a locally closed set and every locally closed set is a B- set. Since regular closed sets are semi-regular and since semi-regular sets are semi-closed, the following implications are obvious:

$$
A-\text { set } \Rightarrow A B-\text { set } \Rightarrow B-\text { set, }
$$

but none of them of course is reversible [4]. Moreover, since the intersection of an open set and a semi-regular set is always semi-open, every $A B$-set is semi-open.

A space $(X, \tau)$ is called a partition space if every open subset of $X$ is closed, see [4]. $(X, \tau)$ is called hyper connected if every open subset of $X$ is dense. $(X, \tau)$ is called extremely disconnected (simply, ED) if every open subset of $X$ has an open closure or equivalently if every regular closed set 
is open. Spaces that contain two disjoint dense subsets are called resolvable. $(X, \tau)$ is called strongly irresolvable [6] if every open subspace of $X$ is irresolvable, i.e. it can not be represented as a disjoint union of two dense subsets. In [5], it has been pointed out that a space is strongly irresolvable if and only if every preopen set is semi-open. $(X, \tau)$ is said to be P-closed [3] (resp., quasi-H-closed (simply, QHC)) if every preopen (resp., open ) cover of $X$ has a finite subfamily the preclosures (resp., closures) of whose members cover $X .(X, \tau)$ is said to be strongly compact [10] if every preopen cover has a finite subcover. $(X, \tau)$ is called nearly compact [13] if every cover of $X$ by regular open sets has a finite sub cover. Thompson [15] introduced the class of $S$-closed spaces, where a space $X$ is called S-closed if every semi-open cover of $X$ has a finite subfamily the closures of whose members cover $X$, or equivalently, if every regular closed cover of $X$ has a finite subcover. $(X, \tau)$ is said to be strongly p-regular [5] (resp., p-regular, almost $p$ - regular) if for each point $x \in X$ and each pre-closed set (resp., closed set, regular closed set) $F$ such that $x \notin F$, there exist disjoint preopen sets $V$ and $U$ such that $x \in U$ and $F \subseteq V$. $(X, \tau)$ is called s-closed, if every semi-open cover has a finite subfamily the semi-closures of whose members cover $X$.

Throughout this paper, $(X, \Im)$ and $(Y, \delta)$ stand for topological spaces with no separation axioms assumed unless otherwise mentioned.

The fundamental notion of generalized open sets was introduced and explored by several authors. In recent years a number of other generalizations of open sets have been studied. Generalized open sets play a very important role in General Topology and they are now the research topics of many topologists worldwide. In fact, a significant theme in General Topology and Real analysis concerns the variously modified forms of continuity, separation axioms etc. by utiliaing generalized open sets. One of 
the most well known notions and also an inspiration source is the notion of generalized preopen sets introduced in [1]. This class is a superset of the class of semi-closed sets, the class of $\alpha$-sets, the class of AB-sets, the class of A-sets and the class of semi-regular sets. Moreover, these investigations lead to solve the problem of finding the continuity dual of some generalized continuous functions in order to have a decomposition of continuity.

This paper is divided as follows: In Section 2, the concept of generalized preopen set is introduced and explored. Properties and connections to other well-known weak and generalized open sets are discussed. Moreover, a characterization of closed and open sets is provided and spaces are studied. In Section 3, generalized preopen compactness is introduced and connections to other types of compactness are discussed. Finally, Section 4 is devoted to discussing new separation axioms defined via generalized preopen sets.

\section{Generalized preopen sets in certain spaces}

We begin this section by recalling the concept of generalized preopen set and some related results from [1].

Definition 1. [1] A subset $A$ of a space $X$ is generalized preopen (simply, gpo-) set if $\operatorname{cl}(A) \subseteq U$, whenever $U$ is a preclosed subset such that $U \supseteq A$. Complements of gpo- sets are called generalized preclosed (simply, gpc-) sets.

By $g p c(X, \tau)$ (resp., $g p c(X, \tau))$, we denote the collection of all gpc (resp., gpc) -sets in $\mathrm{X}$.

Lemma 1. [1] A subset $A$ of a space $X$ is gpc if and only if for every preopen set $V$ contained in $A, V \subseteq \operatorname{int}(A)$. 
We remark that if $A$ and $B$ are gpo- sets, then $A \cap B$ need not be a gpo-set, while arbitrary unions of gpo-sets are gpo-sets.

Example 1. Since (1, 3], as a subspace of the reals with the standard topology, is a gpo- set but not preopen, and $(0,1]$ with the indiscrete topology is preopen but not a gpo- set.

Example 2. Let $X=\{a, b, c, d\}$ and

$$
\tau=\{\phi,\{a\},\{b\},\{a, b\},\{b, c, d\},\{a, c, d\},\{c, d\}, X\} .
$$

Then $X$ is an ED space, $\{c\}$ is a preclosed set that is not a gpo- set. Moreover, $\{c\}$ is not a regular closed set.

Definition 2. A space $(X, \tau)$ is gpo- irresolvable if every preopen set is a gpo- set.

Theorem 1. In a strongly irresolvable space, every semi-open set is a gposet.

Theorem 2. If $(X, \tau)$ is strongly irresolvable, then it is gpo-irresolvable.

Proof: Let $A$ be a preopen set. Then since $X$ is strongly irresolvable, $A$ is semi-open and by Theorem $2, A$ is a gpo- set.

The converse of Theorem 2 is not true in general. $A=(-\infty, 1]$ with the leftray topology on the reals is a gpo- set and a preopen set but not semi-open.

The following is a new characterization of open sets.

Theorem 3. [1] $A$ subset $A \subseteq X$ is open if and only if $A$ is a gpo-set and a preopen set. 


\section{Gpo- compact spaces}

Several types of compact spaces were discussed in $[3,4,5,8,11]$. In this section, gpo-compact notion is introduced and connections to other several well-known types of compactness are discussed.

Definition 3. A space $(X, \tau)$ is gpo-compact if every gpo-cover (a cover consisting of gpo- sets) of $X$ has a finite subcover.

Equivalently, $(X, \tau)$ is gpo-compact if every gpo-cover of $X$ has a finite subcover. A submaximal space is an example of a gpo-compact space. The proof of the following result follows from the fact that every open set is a gpo-set.

Theorem 4. If $(X, \tau)$ is a gpo-compact space, then it is compact.

Since every compact space is nearly compact and a QHC-space, a gpocompact space is nearly compact and QHC.

Theorem 5. If a space $(X, \tau)$ is gpo-irresolvable and gpo-compact, then it is strongly compact.

Proof: Let $\mathbf{A}=\left\{A_{\alpha}: \alpha \in \Delta\right\}$ be a preopen-cover of $X$. Then since $X$ is gpo- irresolvable, by Theorem $1, \mathbf{A}$ is a gpo- cover of $X$ and since $X$ is gpo-compact, it has a finite subcover. Thus $X$ is strongly compact.

The converse of Theorem 4 need not be true since a gpo- set need not be preopen. In addition, the notions of gpo-irresolvable and gpo-compact are independent.

Corollary 1. If a space $(X, \tau)$ is gpo-irresolvable and gpo-compact, then it is P-closed. 
Proof: Let $\mathbf{A}=\left\{A_{\alpha}: \alpha \in \Delta\right\}$ be a preopen cover of $X$. Since $X$ is gpo-irresolvable, $A_{\alpha}$ is a gpo-set for all $\alpha \in \Delta$ and $\mathbf{A}$ is a gpo-cover of $\mathrm{X}$. Since $X$ is gpo-compact, it has a finite subcover. Thus $X \subseteq \bigcup_{i=1}^{n} A_{\alpha i}$. But $\bigcup_{i=1}^{n} A_{\alpha i} \subseteq \bigcup_{i=1}^{n} \operatorname{pcl}\left(A_{\alpha i}\right)$, and so $X$ is P-closed.

Since a gpo- set need not be preopen, a P-closed space need not be gpo-irresolvable or gpo-compact.

Corollary 2. If $(X, \tau)$ is P-closed, $T_{0}$ and gpo-compact, then it is strongly compact.

Proof: By Theorem 3, $(X, \tau)$ is strongly irresolvable. By Theorem 2, $(X, \tau)$ is gpo-irresolvable and by Theorem $5,(X, \tau)$ is strongly compact.

Theorem 6. If $(X, \tau)$ is a gpo-compact space, then it is s-closed.

Proof: Let $\mathbf{A}=\left\{A_{\alpha}: \alpha \in \Delta\right\}$ be a semi-open cover of $\mathrm{X}$. Then $A$ is a gpo-cover of $X$. Since $X$ is gpo-compact, it has a finite subcover such that $X \subseteq \bigcup_{i=1}^{n} A_{\alpha i}$. But $\bigcup_{i=1}^{n} A_{\alpha i} \subseteq \bigcup_{i=1}^{n} \operatorname{scl}\left(A_{\alpha i}\right)$. Thus $X \subseteq \bigcup_{i=1}^{n} \operatorname{scl}\left(A_{\alpha i}\right)$, so $X$ is s-closed.

Since an s-closed space is S-closed, a gpo-compact space is S-closed.

Theorem 7. If a map $f:(X, \tau) \rightarrow(Y, \sigma)$ is gpo-irresolute (resp., gpocontinuous) surjective and $K$ is gpo-compact subset of $X$, then $f(K)$ is gpo-compact (resp, QHC) in $Y$.

Proof: Let $\mathbf{A}=\left\{A_{\alpha}: \alpha \in \Delta\right\}$ be any gpo- (open) cover of $f(K)$. Since $f$ is gpo-irresolute (resp, gpo-continuous), $A^{*}=\left\{f^{-1}\left(A_{\alpha}\right): \alpha \in \Delta\right\}$ is gpocover of $K$ where $K$ is gpo-compact. Thus it has a finite subcover. That is 
$K \subseteq \bigcup_{i=1}^{n} f^{-1}\left(A_{\alpha i}\right)$. Since $f$ is gpo-irresolute (gpo-continuous) and surjective, we have $f(K) \subseteq \bigcup_{i=1}^{n} f\left(f^{-1}\left(A_{\alpha i}\right)\right) \subseteq \bigcup_{i=1}^{n} A_{\alpha i}$ (resp., $f(K) \subseteq \bigcup_{i=1}^{n} A_{\alpha i} \subseteq$ $\left.\bigcup_{i=1}^{n} c l\left(A_{\alpha i}\right)\right)$. Thus $f(K)$ is gpo-compact (resp., QHC) in $Y$.

Definition 4. A space $(X, \tau)$ is strongly s-compact if for every gpo-cover $\mathbf{A}=\left\{A_{\alpha}: \alpha \in \Delta\right\}$, there exist $A_{\alpha 1}, A_{\alpha 2}, \ldots, A_{\alpha n} \in \mathbf{A}$ such that $X \subseteq$ $\bigcup_{i=1}^{n} \operatorname{cl}\left(A_{\alpha i}\right)$.

If $(X, \tau)$ is gpo-compact, then clearly it is strongly gpo-compact, since $\bigcup_{i=1}^{n} A_{\alpha i} \subseteq \bigcup_{i=1}^{n} \operatorname{cl}\left(A_{\alpha i}\right)$ for every $A_{\alpha i} \subseteq X, i=1,2 \ldots, n$.

Definition 5. A space $(X, \tau)$ is strongly Ogpo-regular if $X$ has a gpocover $\mathbf{A}=\left\{A_{\alpha}: \alpha \in \Delta\right\}$ for all $\mathrm{x} \in X$, and for all $A_{\alpha}(x) \in \mathbf{A}$ such that $x \in A_{\alpha}(x)$, there exists $U_{x} \in g p o(X)$ such that $x \in U_{x} \subseteq c l\left(U_{x}\right) \subseteq A_{\alpha}(x)$.

Theorem 8. If $(X, \tau)$ is strongly gpo-compact and strongly Ogpo-regular, then it is gpo-compact.

Proof: Let $\mathbf{A}=\left\{A_{\alpha}: \alpha \in \Delta\right\}$ be a gpo-cover of $X$. Then since $X$ is strongly Ogpo-regular, for all $x \in X$, there exists $A_{\alpha}(x) \in \mathbf{A}$ such that $x \in$ $A_{\alpha}(x)$ and there exists $U_{x} \in g p o(X)$ with $x \in U_{x} \subseteq \operatorname{cl}\left(U_{x}\right) \subseteq A_{\alpha}(x)$. Thus $\left\{U_{x}: x \in X\right\}$ is a gpo-cover of $X$. Since $X$ is strongly gpo-compact, there exist $U_{x 1}, U_{x 2}, \ldots, U_{x n}$ such that $X \subseteq \bigcup_{i=1}^{n} c l\left(U_{x i}\right) \subseteq \bigcup_{i=1}^{n} A_{\alpha i}(x)$. Therefore $X$ is gpo-compact.

Corollary 3. If $(X, \tau)$ is strongly Ogpo-regular, then it is strongly gpocompact if and only if it is gpo-compact. 
Definition 6. A space $(X, \tau)$ is gpo-regular if for every gpo-cover $\mathbf{A}=$ $\left\{A_{\alpha}: \alpha \in \Delta\right\}$ of $X$ and for every $x \in A_{\alpha}(x) \in \mathbf{A}$, there exists a preopen set $U_{\alpha}$ such that $x \in U_{\alpha} \subseteq A_{\alpha}$.

Theorem 9. If $(X, \tau)$ is a gpo-regular and a strongly compact space, then it is gpo-compact.

Proof: Let $\mathbf{A}=\left\{A_{\alpha}: \alpha \in \Delta\right\}$ be a gpo-cover of $X$. Then since $X$ is gpo-regular, for all $x \in A_{\alpha}(x)$ there exists $U \in p o(X)$ such that $x \in U_{\alpha} \subseteq A_{\alpha}$ for all $\alpha \in \Delta$ and so $\left\{U_{\alpha}: \alpha \in \Delta\right\}$ is a preopen cover of $X$. Since $X$ is strongly compact, then $X=\bigcup_{i=1}^{n} U_{\alpha i} \subseteq \bigcup_{i=1}^{n} A_{\alpha i}$. Therefore $X$ is gpo-compact.

The converse of the preceding Theorem need not be true since a preopen set need not be a gpo- set. Moreover, it is clear that the notions of gpo-regular and gpo-irresolvable spaces are independent. The proof of the following result follows immediately from Theorem 8 and Theorem 9 .

Corollary 4. If a space $(X, \tau)$ is gpo-regular and gpo-irresolvable, then it is gpo-compact if and only if it is strongly compact.

Definition 7. A space $(X, \tau)$ is o-regular if for every gpo-cover $\mathbf{A}=\left\{A_{\alpha}\right.$ : $\alpha \in \Delta\}$ of $X$ and for every $x \in A_{\alpha}(x) \in \mathbf{A}$, there exists an open set $U$, such that $x \in U_{x} \subseteq \operatorname{cl}\left(U_{x}\right) \subseteq A_{\alpha}(x)$.

Theorem 10. If $(X, \tau)$ is o-regular, then it is gpo-compact if and only if it is $Q H C$.

Proof: Let $(X, \tau)$ be gpo-compact. By Theorem $7,(X, \tau)$ is a compact space, so it is QHC-space. 
Conversely, let $\mathbf{A}=\left\{A_{\alpha}: \alpha \in \Delta\right\}$ be a gpo- cover of $X$. Since $X$ is o-regular, for every $x \in A_{\alpha}(x) \in \mathbf{A}$, there exists an open set $\mathrm{U}$ such that $x \in U_{x} \subseteq \operatorname{cl}\left(U_{x}\right) \subseteq A_{\alpha}(x)$. Thus $\left\{U_{\alpha}: \alpha \in \Delta\right\}$ is an open cover of $X$. Since $X$ is QHC, there exists a finite family such that $X=\bigcup_{i=1}^{n} \operatorname{cl}\left(U_{\alpha i}\right) \subseteq \bigcup_{i=1}^{n} A_{\alpha i}$. Hence $(X, \tau)$ is gpo-compact.

Corollary 5. If $(X, \tau)$ is o-regular, then it is gpo-compact if and only if it is s-closed( $S$-closed, compact, nearly compact).

Proof : Clearly s-closed ( S-closed, compact, nearly compact) imply QHC and by Theorem 10, QHC imply gpo-compact.

Conversely, if $(X, \tau)$ is gpo-compact, then by Theorem 7 it is compact and so it is nearly compact and by Theorem 12, it is s-closed. Thus it is S-closed.

Theorem 11. If $(X, \tau)$ is o-regular, then it is $Q H C$ if and only if it is strongly gpo-compact.

Proof: Let $\mathbf{A}=\left\{A_{\alpha}: \alpha \in \Delta\right\}$ be a gpo-cover of $X$. Since $X$ is oregular, for every $x \in A_{\alpha}(x) \in \mathbf{A}$ there exists an open set $U$ such that $x \in U_{x} \subseteq \operatorname{cl}\left(U_{x}\right) \subseteq A_{\alpha}(x)$. So $\left\{U_{\alpha}: \alpha \in \Delta\right\}$ is an open cover of X. Since $X$ is QHC, $X \subseteq \bigcup_{i=1}^{n} c l\left(U_{x}\right) \subseteq \bigcup_{i=1}^{n} A_{\alpha i}(x)$. Since $\bigcup_{i=1}^{n} A_{\alpha i} \subseteq \bigcup_{i=1}^{n} c l\left(A_{\alpha i}\right)$ for every $A_{\alpha i} \subseteq X, i=1,2 \ldots, n ., X$ is strongly gpo- compact.

Conversely, let $\mathbf{A}=\left\{A_{\alpha}: \alpha \in \Delta\right\}$ be an open cover of $X$. Then $\mathbf{A}$ is a gpo- cover of $X$ and since $X$ is strongly gpo-compact, $X \subseteq \bigcup_{i=1}^{n} \operatorname{cl}\left(A_{\alpha i}(x)\right)$. Thus $X$ is QHC.

\section{Separation axioms via gpo-sets}

In this section, several new separation axioms via gpo- sets are introduced. 
Connections to other well-known ones are also discussed.

Definition 8. Let $(X, \tau)$ be a topological space. Then

(a) $(X, \tau)$ is called a $\mathbf{T}_{\mathbf{p} \circ}$ - space if for each pair of distinct points $x, y \in X$, there is either a gpo- set containing $\mathrm{x}$ but not $\mathrm{y}$ or a gpo- set containing $y$ but not $\mathrm{x}$.

(b) $(X, \tau)$ is called a $\mathbf{T}_{\mathbf{p} \mathbf{1}}$ - space if for each pair of distinct points $x, y \in X$, there is a gpo- set containing $\mathrm{x}$ but not $\mathrm{y}$, and a gpo- set containing $y$ but not $x$.

(c) $(X, \tau)$ is called a $\mathbf{T}_{\mathbf{p} \mathbf{2}}$ - space if for each pair of distinct points $x, y \in X$, there exist gpo- sets $U$ and $V$ such that $x \in U, y \in V$ and $U \cap V=\phi$.

(d) $(X, \tau)$ is called a weak regular space if for each closed subset $F \subset X$ and each point $x \notin F$, there exist gpo- sets $U$ and $V$ such that $x \in U, F \subset V$ and $U \cap V=\phi$. A weak regular $T_{p 1}-$ Space is called $\mathbf{T}_{\mathbf{p} \mathbf{3}}$ - space.

(e) $(X, \tau)$ is called a weak normal space if for each pair of disjoint closed subsets $F_{1}$ and $F_{2}$ of $X$ there exist gpo-sets $U$ and $V$ such that $F_{1} \subset U, F_{2} \subset V$ and $U \cap V=\phi . A$ weak normal $T_{p 1}-$ Space is called a $\mathbf{T}_{\mathbf{p} 4}$ - space.

It is clear that the $T_{p 2}$ condition implies the $T_{p 1}$ condition, which in turn implies the $T_{p o}$ condition. Since the notions of open set and gpo-set are independent, weak regularity and regularity are also independent notions.

Theorem 12. If each point $x \in X$ is a gpo-set, then the space $(X, \tau)$ is a $T_{p 1}-$ Space.

Proof: Let each point of $X$ be a gpo subset of X. If $|X|=1$, the result is clear. Thus let $x$ and $y$ be distinct points of $X$. Then $\{y\}$ is a gpo-set, so $X \backslash\{y\}$ is a gpo-set containing $x$ but not y. It follows that $(X, \tau)$ is a $T_{p 1}-$ Space. 
Definition 9. A space $X$ is called completely p-regular if for each closed set $A \subset X$ and each $p \notin A$, there exists a gp- continuous map $f: X \rightarrow I$ such that $f(p)=1$ and $f(A)=0$. A completely $p$ - regular $T_{p 1}-$ Space is called a $\mathbf{T}_{\mathbf{p} 3 \frac{1}{2}}-$ Space.

Definition 10. A space $(X, \tau)$ is called completely p- normal if given any two disjoint subsets $A$ and $B$ of $X$, there exist disjoint gpo- sets $U$ and $V$ of $X$ such that $A \subset U$ and $B \subset V$. A completely $p$ - normal $T_{p 1}-$ Space is called a $\mathbf{T}_{\mathbf{p} 5}$ - Space.

The proof of the following results follow from the fact that open sets are gpo-sets.

Theorem 13. Let $(X, \tau)$ be a space. Then every $T_{i}-$ Space is a $T_{p i}-$ space, for $i=0,1,2,3,3 \frac{1}{2}, 4,5$.

Theorem 14. Every $T_{p 3 \frac{1}{2}}-$ Space is a $T_{p 3}-$ Space and every $T_{p 5}-$ Space is a $T_{p 4}-$ Space.

\section{References}

[1] Al-Hawary, Talal, Generalized Preopen Sets, Questions Answers Gen. Topology 29 (1), pp. 73-80, (2011).

[2] Andrijevi'c, D., Semi-preopen sets, Mat. Vesnik. 38, pp. 24-32, (1986).

[3] Abo-Khadra, A., On Generalized Forms of Compactness, Masters Thesis, Tanta University (Egypt, 1989). 
[4] Dontchev, J. and Helsinki, J., Between A- and B-sets, Acta Math. Hung. 69(1-2), pp. 111-122, (1998).

[5] Dontchev, J., Ganster, M.and Noiri, T., On P-closed Spaces, Inter. J. Math. Math. Sci. 24 (3), pp. 203-212, (1998).

[6] Foran, J. and Liebnitz, P., A Characterization of Almost Resolvable Spaces, Rand Circ. Mat. Palermo (2), pp. 136-141, (1991).

[7] Ganster, M. and Reilly, I.L.., A Decomposition of Continuity, Acta Math. Hungar, 56, no 3-4, pp. 299-301, (1990).

[8] Jankovic, D., Reilly, I.L. and Vamanamurthy M., On Strongly Compact Topological Spaces, Acta Math. Hung., pp. 29-40, (1988).

[9] Levine, N., Generalized Closed sets in Topology, Rend. Circ. Mat. Palermo (2) 19, pp. 89-96, (1970).

[10] Mashhour, A. S., Abd EL-Monsef, M. E., Hasainen, I. A. and Noiri, T., Strongly Compact Spaces, Delta J. Sci, pp. 30-46, (1984).

[11] Mashhour, A. S., Abd EL-Monsef, M. E. and ElDeep, S. N., On Pre-continuous and Weak Pre-continuous Mappings, Proc. Math. and Phys. Soc. Egypt, 53, pp. 47-53, (1982).

[12] Mashhour, A. S., Abd EL-Monsef, M. E. and ElDeep, S. N., $\alpha$-continuous and $\alpha$-open Mappings, Acta Math. Hung., 3, pp. 213218, (1982).

[13] Mathur, A. and Singal, M. K., On Nearly-Compact Spaces, Boll. Un. Mat. Ital., pp. 702-710, (1969).

[14] Reilly, I. L. and Vamanamurthy, M., On $\alpha$-continuity in Topological Spaces, J. Indian Acad. Math., 18, pp. 89-99, (1996). 
[15] Thompson, T., S-closed spaces, Proc. Amer. Math. Soc., pp. 335-338, (1971).

[16] Tong, J., A Decomposition of Continuity, Acta Math. Hungar., 48, pp. 11-15, (1986).

\section{Talal Al-Hawary}

Department of Mathematics

Yarmouk University

Irbid-Jordan

e-mail : talalhawary@yahoo.com 